\title{
Multimodal approach to portal hypertension and gastric varices before hepatic resection for hepatocellular carcinoma: a case report
}

Norifumi Harimoto ${ }^{1 *}$ (D) Kenichiro Araki ${ }^{1}$, Ryo Muranushi ${ }^{1}$, Kouki Hoshino ${ }^{1}$, Kei Hagiwara ${ }^{1}$, Norihiro Ishii ${ }^{1}$, Mariko Tsukagoshi ${ }^{1,2}$, Takamichi Igarashi ${ }^{1}$, Akira Watanabe ${ }^{1}$, Norio Kubo ${ }^{1}$, Kei Shibuya ${ }^{3}$, Masaya Miyazaki ${ }^{4}$, Hirofumi Kawanaka ${ }^{5}$ and Ken Shirabe ${ }^{1}$

\begin{abstract}
Background: Liver cirrhosis occurs in approximately $80-90 \%$ of patients with hepatocellular carcinoma (HCC), and hepatic resection may be dangerous because of well-documented liver cirrhosis, which may be accompanied by portal hypertension (PH). Here we report a patient with advanced HCC with gastric varices and $\mathrm{PH}$ who experienced a good clinical course after undergoing balloon-occluded retrograde transvenous obliteration (BRTO), percutaneous transhepatic portal vein embolization (PTPE), hand-assisted laparoscopic (HALS) splenectomy, and right lobectomy of the liver.
\end{abstract}

Case presentation: A 72-year-old man had two HCCs with gastric varices. CT revealed one tumor $(4.5 \mathrm{~cm})$ located in segment 7, involving the right hepatic vein, adjacent to the middle hepatic vein. Another tumor $(2.7 \mathrm{~cm})$ was located in segment 6 . He first underwent BRTO for gastric varices and PTPE for planned right lobectomy of the liver. To reduce PH, HALS splenectomy was performed, and uncomplicated right lobectomy of the liver was performed 10 weeks after the first visit. He has remained free of recurrence for at least 1 year.

Conclusions: Our patient underwent uncomplicated BRTO, PTPE, HALS splenectomy, and right lobectomy of the liver for advanced HCC with PH. Controlling portal pressure is important when hepatic resection is required to treat $\mathrm{HCC}$ with $\mathrm{PH}$.

Keywords: Hepatocellular carcinoma, Portal hypertension, Portal vein embolization, Balloon-occluded retrograde transvenous obliteration, Hepatic resection

\section{Background}

Hepatocellular carcinoma (HCC) is the third most common malignancy worldwide [1]. Liver cirrhosis occurs in approximately $80-90 \%$ of patients with HCC, and hepatic resection tends to be dangerous because of liver cirrhosis [2, 3] if patients with liver cirrhosis have

\footnotetext{
* Correspondence: nharimotoh1@gunma-u.ac.jp

'Department of Hepatobiliary and Pancreatic Surgery, Graduate School of Medicine, Gunma University, 3-39-22, Showamachi, Maebashi 371-8511, Japan

Full list of author information is available at the end of the article
}

concomitant portal hypertension (PH). $\mathrm{PH}$ is pathophysiologically characterized by thrombocytopenia caused by splenomegaly, esophagogastric varices, and portosystemic shunt. In the Barcelona Clinic Liver Cancer (BCLC) staging classification, $\mathrm{PH}$ is identified as a contraindication for hepatic resection because of high postoperative morbidity and poor survival $[2,4,5]$. Patients with $\mathrm{HCC}$ with $\mathrm{PH}$ may be candidates for liver resection because of the safety of the procedure as well as prophylactic perioperative management that may help 
overcome complications after hepatic resection associated with $\mathrm{PH}[6]$.

Here we report a patient with a good clinical course who underwent balloon-occluded retrograde transvenous obliteration (BRTO), percutaneous transhepatic portal vein embolization (PTPE), hand-assisted laparoscopic (HALS) splenectomy, and right lobectomy of the liver for advanced $\mathrm{HCC}$ with $\mathrm{PH}$.

\section{Case presentation}

A 72-year-old man, who was diagnosed with chronic hepatitis $\mathrm{C}$ virus (HCV) infection, was prescribed directacting antivirals. After 6 months of a sustained virological response (SVR), ultrasound (US) imaging detected two liver tumors. Computed tomography (CT) revealed one tumor $(4.5 \mathrm{~cm}$ ) located at segment 7 (Fig. 1a), which involved the right hepatic vein (Fig. 1b) and was adjacent to the middle hepatic vein. A second tumor $(2.7 \mathrm{~cm})$ was located at segment 6 . Contrast-enhanced (CE) CT detected the tumors during the early phase as well as a perfusion defect during the portal phase. Magnetic resonance imaging (MRI) revealed the same features (Fig. 2a, b). CE-CT detected gastric varices as an enlarged gastrorenal shunt from the left renal vein to the left gastric vein (Fig. 3a). Furthermore, endoscopic images showed markedly enlarged nodular (F3), risky gastric varices (Fig. 3b) [7]. ${ }^{18}$ F-fluorodeoxyglucose (FDG) positron emission tomography (PET) revealed the accumulation of ${ }^{18} \mathrm{~F}$-FDG. The maximum standard uptake values were 5.43 at S7 and 4.42 at S6, and distant metastasis was not detected.

Laboratory results were as follows: white blood cell count, $3200 / \mu \mathrm{L}$; platelet count, $75,000 / \mu \mathrm{L}$; total serum albumin, $3.5 \mathrm{~g} / \mathrm{dL}$; \%prothrombin time, 95\%; total serum bilirubin, $0.6 \mathrm{mg} / \mathrm{dL}$; direct bilirubin, $0.03 \mathrm{mg} / \mathrm{dL}$; aspartate aminotransferase, $22 \mathrm{U} / \mathrm{L}$; alanine aminotransferase, $15 \mathrm{U} / \mathrm{L} ;$ alkaline phosphatase, $191 \mathrm{U} / \mathrm{L}$; gammaglutamyltranspeptidase, $49 \mathrm{U} / \mathrm{L}$; ammonia concentration, $51 \mu \mathrm{g} / \mathrm{dL}$; and the indocyanine green dye retention test at $15 \mathrm{~min}$ (ICGR15) was $15.3 \%$. The concentrations of tumor markers such as carcinoembryonic antigen (CEA) and carbohydrate antigen (CA) 19-9 were normal, and the concentrations of arufa-fetoprotein (AFP) $(217.2 \mathrm{ng} /$ $\mathrm{mL}$ ) and des-gamma-carboxy prothrombin (DCP) (1951 $\mathrm{AU} / \mathrm{mL}$ ) were elevated. Hepatitis B virus (HB)s-antigen, $\mathrm{HBc}$-antibody, and HCV-RNA were undetectable, and $\mathrm{HCV}$-antibody was positive. Mac-2 binding protein glycosylation isomer (M2BPGi) which indicated liver fibrosis was 1.95 cut off index (COI). Virtual touch quantification (VTQ) using ultrasonography was 3.09 $\mathrm{mL} / \mathrm{s}$. The Child-Pugh score was 6, grade A. The Albumin-Indocyanine Green Evaluation (ALICE) score was - 1.731 and ALICE grade was $2 b$.

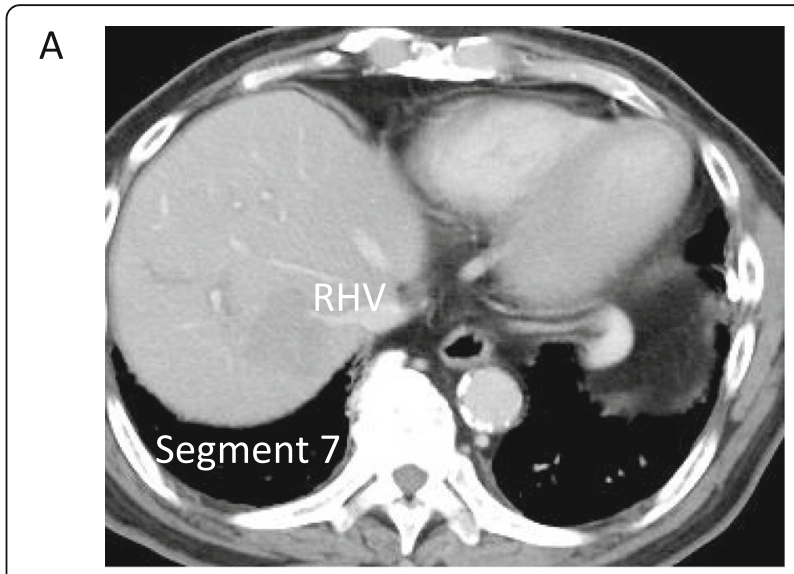

B

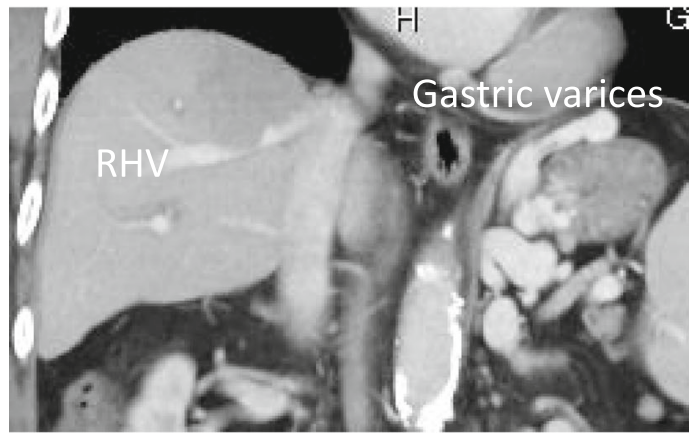

C

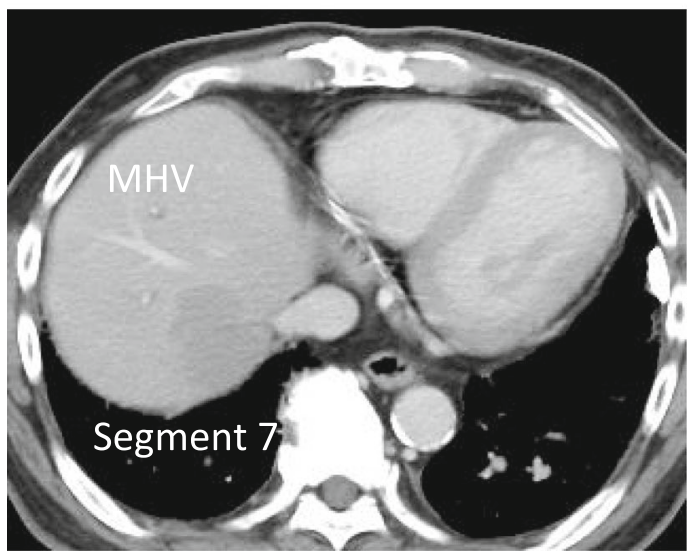

Fig. 1 CT imaging of HCC. CT showing the tumor located in segment 7 involving the right hepatic vein, $\mathbf{a}$ transverse plane, $\mathbf{b}$ coronal plane, and c close to the middle hepatic vein. HCC, hepatocellular carcinoma; CT, computed tomography

The patient was administered insulin injection therapy because of diabetes mellitus, and he underwent percutaneous coronary intervention for coronary artery stenosis in 2015. He was diagnosed with stage III HCC with risky gastric varices. We first performed BRTO for gastric varices (Fig. 3c). The wedged hepatic venous pressure (WHVP) was 13 $\mathrm{mmHg}$, and the hepatic venous pressure gradient (HVPG) was $11 \mathrm{mmHg}$. WHVP increased to 15 mmHg after BRTO. The tumor was close to the 

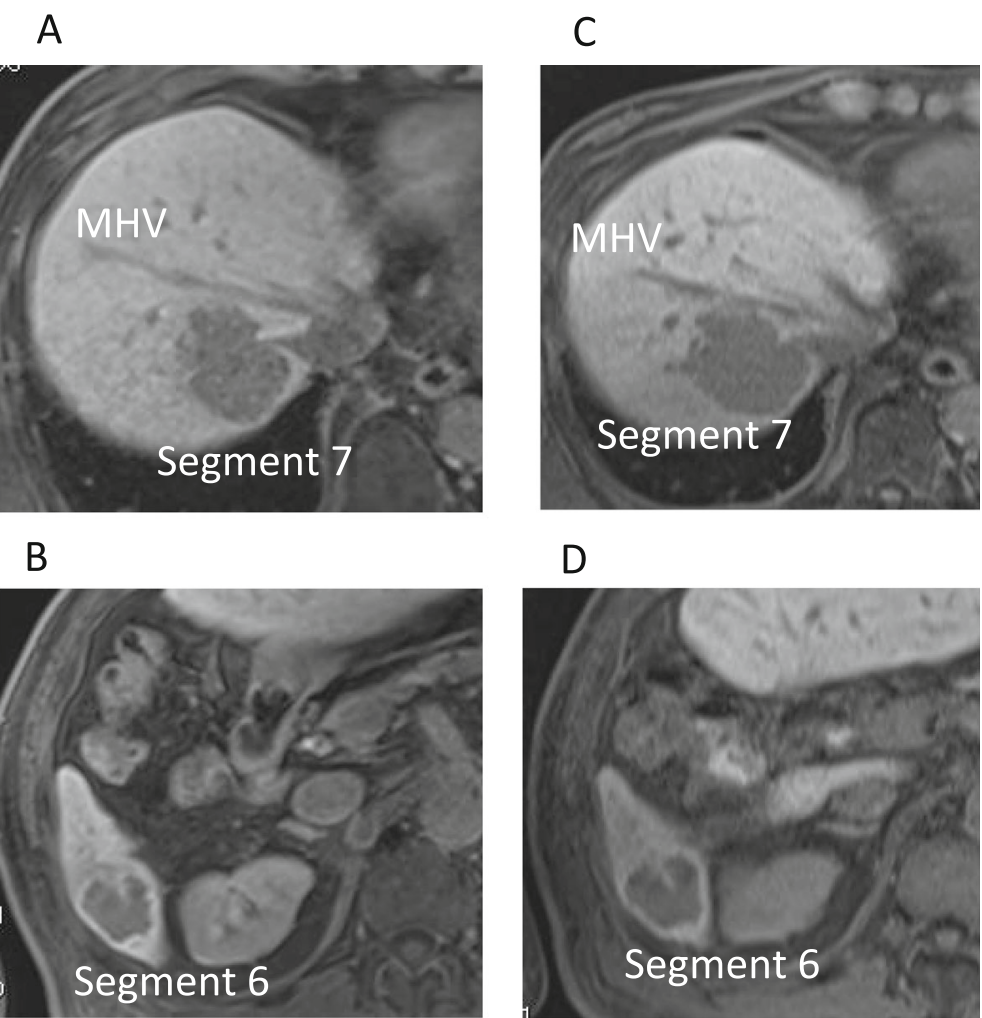

Fig. 2 MRI imaging of HCC. a MRI showing the tumor located in segment 7 at the first visit. b MRI shows the tumor located in segment 6 at the first visit. c MRI shows the tumor located in segment 7 before hepatic resection. The tumor grew to $5.1 \mathrm{~cm} 10$ weeks after the first visit. $\mathbf{d}$ MRI showing the tumor located in segment 6 before hepatic resection. The tumor grew to $3.1 \mathrm{~cm} 10$ weeks after the first visit. MRI, magnetic resonance image; HCC, hepatocellular carcinoma; $C T$, computed tomography

middle hepatic vein, and we therefore planned righthepatic resection of the liver.

The remnant liver volume was $867 \mathrm{~mL}$ (59.4\%), according to 3D-CT volumetry (Fig. 4a). Although the remnant liver volume was sufficient, to reduce surgical risk, we planned PTPE and HALS splenectomy before hepatic resection because of PH. We performed PTPE 9 days after BRTO without complications. After PTPE, the portal vein pressure was $15 \mathrm{mmHg}$. Spleen volume was $428 \mathrm{~g}$ at the first visit (Fig. 4b) and increased to $512 \mathrm{~g}$ just before splenectomy. Three weeks after PTPE, HALS splenectomy was performed using an $8-\mathrm{cm}$ midline incision and four ports. The spleen weighed $439 \mathrm{~g}$. Operation time was $191 \mathrm{~min}$, blood loss was $42 \mathrm{~g}$, and blood transfusion was not required. In this patients was received anti-thrombin III (ATIII) concentrates for 3 days after splenectomy because of low ATIII activity as a prophylactic treatment. There was no portal vein thrombosis, and the patient was discharged 10 days after splenectomy without complications.

Right hepatic resection was performed 27 days after HALS splenectomy. ICGR15 improved just before hepatectomy (Table 1). Three-dimensional-CT revealed an increase in the remnant liver volume (1097 $\mathrm{mL}, 64.2 \%)$.
Immediately before hepatic resection, WHVP was 13 $\mathrm{mmHg}$ and HVPG was $9 \mathrm{mmHg}$. M2BPGi increased to 2.36 COI. The sizes of the tumors slightly increased (5.1 $\mathrm{cm}$ at $\mathrm{S} 7$ and $3.1 \mathrm{~cm}$ at S6) (Fig. 2c, d), and the levels of tumor markers increased (AFP, $611 \mathrm{ng} / \mathrm{mL}$ and DCP, $3289 \mathrm{AU} / \mathrm{mL}$ ). Operation time was $473 \mathrm{~min}$, blood loss was $136 \mathrm{~g}$, and a blood transfusion was not required.

Figure 5 shows the macroscopic findings of the resected tumors. The right hepatic vein was surrounded by the tumor, although a venous thrombus was not detected. The patient was discharged 13 days after hepatic resection without complications. Histological diagnosis was well- to moderately differentiated HCC, and portal vein invasion by the tumor was detected in S7. Fibrosis grade was F3. Table 1 summarizes the patient's clinical course. When this manuscript was submitted, he was free of recurrence for 1 year. He received vaccination after hepatectomy to prevent over whelming post splenectomy infection (OPSI).

\section{Discussion}

To control PVP, it is important to consider the strategy for HCC with PH. Berzigotti et al. [8] determined portal hypertension as an independent factor for decreased longterm survival and increased perioperative decompensation 


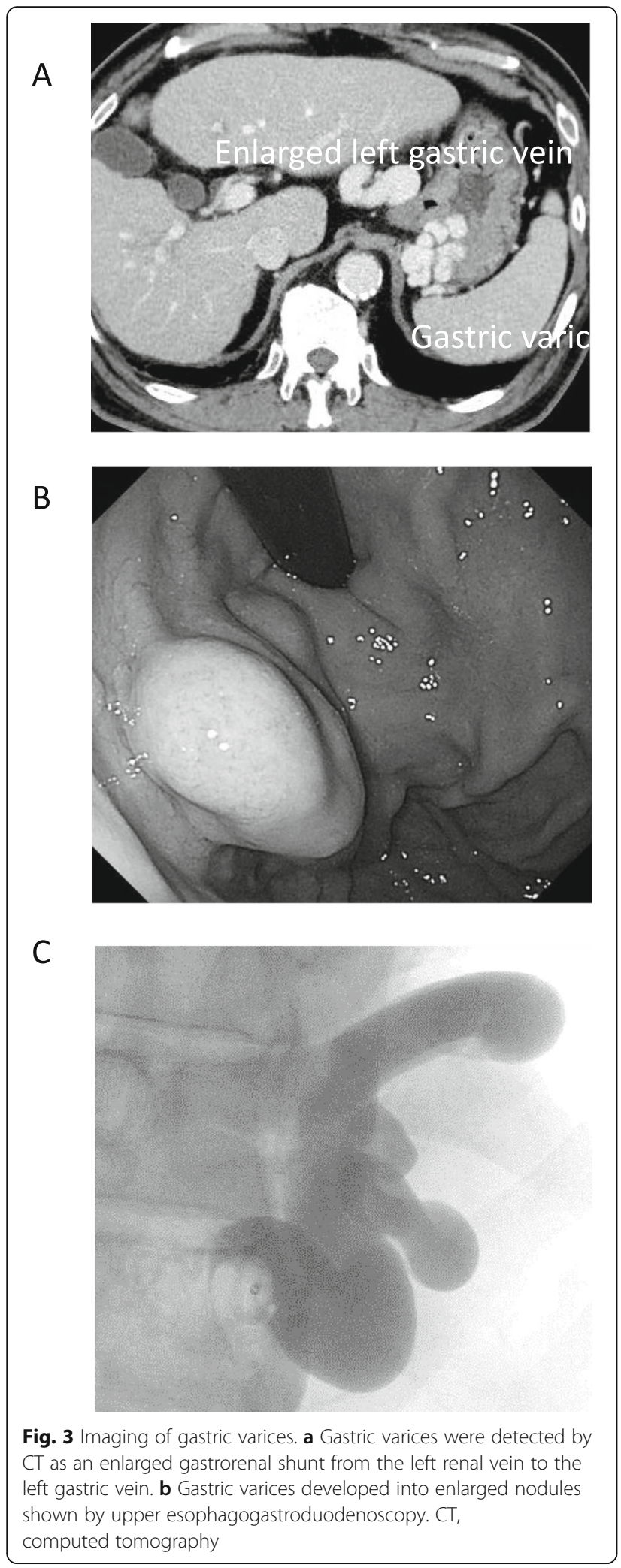

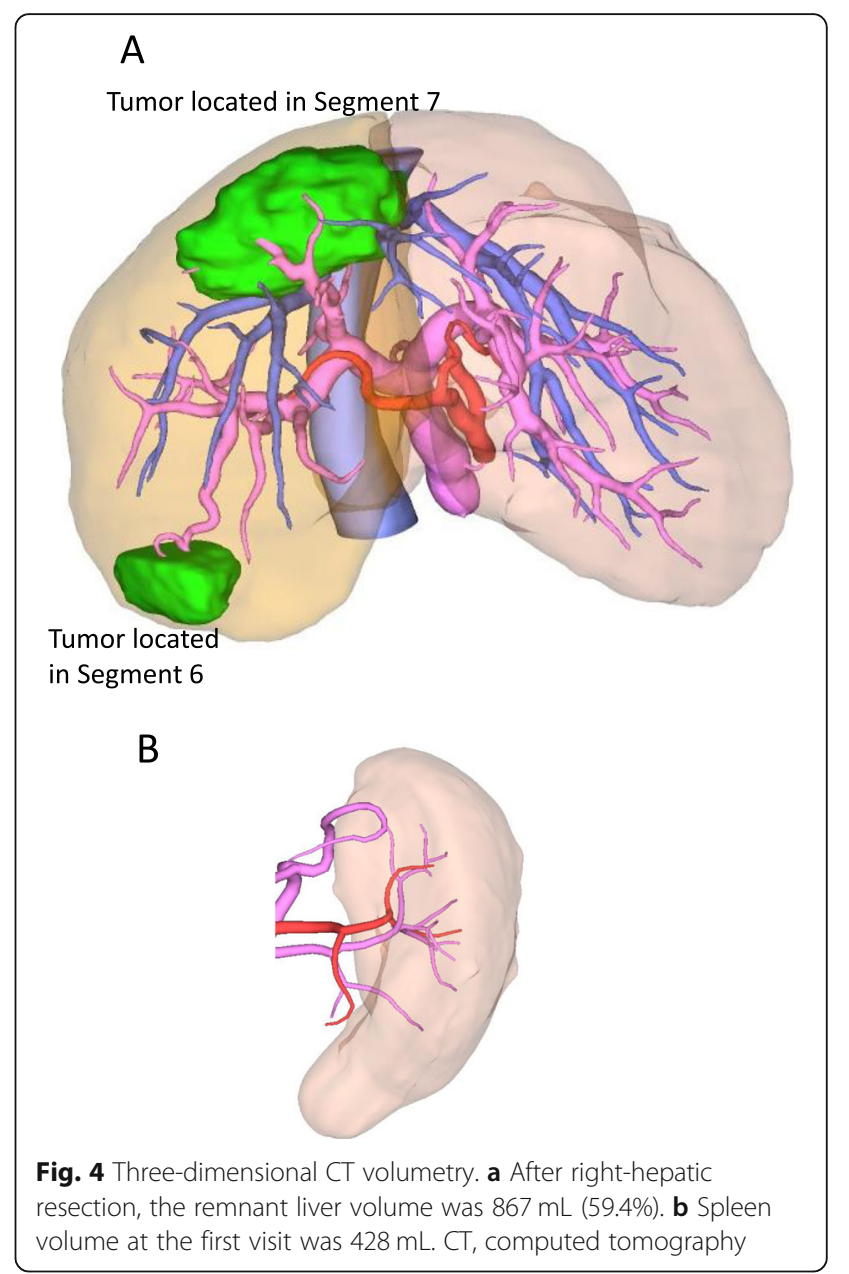

after resection of HCC. Bruix and Llovet evaluated the role of direct preoperative measurement of portal venous pressure to predict the outcome after liver resection in patients with cirrhosis [2, 5]. These studies found that HVPG $\geq 10 \mathrm{mmHg}$ is a predictive factor for postoperative liver decompensation. Furthermore, HVPG is an indirect measurement of the actual PVP, which allows obviating risks linked with the direct puncture of the portal vein.

Evidence indicates that PVP reflects poor prognosis. For example, Chen et al. found that PVP $>16 \mathrm{~cm} \mathrm{H}_{2} \mathrm{O}$ $(=11.8 \mathrm{mmHg})$ with sensitivities of $82 \%$ and specificity of $70 \%$ for predicting postoperative liver failure [9]. Hidaka et al. found that the 5-year overall survival rate and recurrence-free survival rate were significantly higher for patients with low PVP $\left(<20 \mathrm{~cm} \mathrm{H}_{2} \mathrm{O}=14.7\right.$ $\mathrm{mmHg})$ compared with those with high-PVP $(>20 \mathrm{~cm}$ $\mathrm{H}_{2} \mathrm{O}=14.7 \mathrm{mmHg}$ ) [10]. In the present case, we reduced HVPG to $>10 \mathrm{mmHg}$ before hepatic resection. Sirata et al reported that in the ALICE grade 2 group, patients with $\mathrm{PH}$ showed high incidence of large-volume ascites and post-hepatectomy liver failure for HCC [11]. In addition, sectoriectomy or more was also a risk factor to 
Table 1 The summary of clinical course

\begin{tabular}{|c|c|c|c|c|c|c|c|c|}
\hline \multirow[t]{3}{*}{ Progress from the first visit 0} & \multicolumn{2}{|l|}{14 days } & \multicolumn{2}{|c|}{23 days } & \multicolumn{2}{|l|}{44 days } & \multicolumn{2}{|c|}{71 days } \\
\hline & \multicolumn{2}{|l|}{ BRTO } & \multicolumn{2}{|l|}{ PTPE } & \multicolumn{2}{|c|}{ HALS splenectomy } & \multicolumn{2}{|c|}{ Right lobectomy } \\
\hline & Before & After & Before & After & Before & After & Before & After \\
\hline Albumin (g/dL) & 3.5 & 3.4 & 3.4 & 3.4 & 3.4 & 3.2 & 3.4 & 3.5 \\
\hline Total bilirubin (mg/dL) & 0.6 & 0.6 & 0.5 & 0.6 & 0.5 & 0.6 & 0.6 & 0.7 \\
\hline$\% \mathrm{PT}$ & 95 & 102 & 98 & 102 & 104 & 108 & 105 & 92 \\
\hline ICGR15(\%) & 15.1 & 5.2 & - & - & - & - & 12.1 & - \\
\hline ALICE score & -1.73 & -1.96 & - & - & - & - & -1.72 & - \\
\hline ALICE grade & $2 b$ & $2 a$ & - & - & - & - & $2 b$ & - \\
\hline Platelet count $\left(\times 10^{4} / \mu \mathrm{L}\right)$ & 7.6 & 5.7 & 6.5 & 7.9 & 7.2 & 16.3 & 13.8 & 13.9 \\
\hline $\mathrm{NH}_{3}(\mu \mathrm{g} / \mathrm{dL})$ & 51 & 30 & 41 & 48 & 39 & 35 & 35 & 39 \\
\hline $\operatorname{AFP}(\mathrm{ng} / \mathrm{mL})$ & 217.2 & - & - & - & - & - & 611 & 5.8 \\
\hline PIVKAll (mAU/mL) & 1951 & - & - & - & - & - & 3289 & 24 \\
\hline Remnant liver volume $(\mathrm{mL})$ & 867 & - & - & - & - & - & 1097 & - \\
\hline Remnant liver volume (\%) & 59.4 & - & - & - & - & - & 64.2 & - \\
\hline WHVP (mmHg) & 13 & 15 & - & - & - & - & 13 & - \\
\hline HVPG (mmHg) & 11 & 13 & - & - & - & - & 9 & - \\
\hline PVP (mmHg) & - & - & 13 & 15 & - & - & - & - \\
\hline M2BPGi (COI) & 1.95 & - & - & - & - & - & 2.36 & - \\
\hline VTQ $(\mathrm{m} / \mathrm{s})$ & 3.09 & - & - & - & - & - & - & - \\
\hline
\end{tabular}

BRTO balloon-occluded retrograde transvenous obliteration, PTPE percutaneous trans-hepatic portal vein embolization, HALS hand-assisted laparoscopic, PT prothrombin time, ALICE Albumin-Indocyanine Green Evaluation, ICGR15 indocyanine green dye retention test at 15 min, AFP a-fetprotein, DCP des- $\gamma$-carboxy prothrombin, WHVP wedged hepatic venous pressure, HVPG hepatic venous pressure gradient, $P V P$ portal vein pressure, M2BPGi Mac-2 binding protein glycosylation isomer, COI cut-off index, VTQ virtual touch quantification

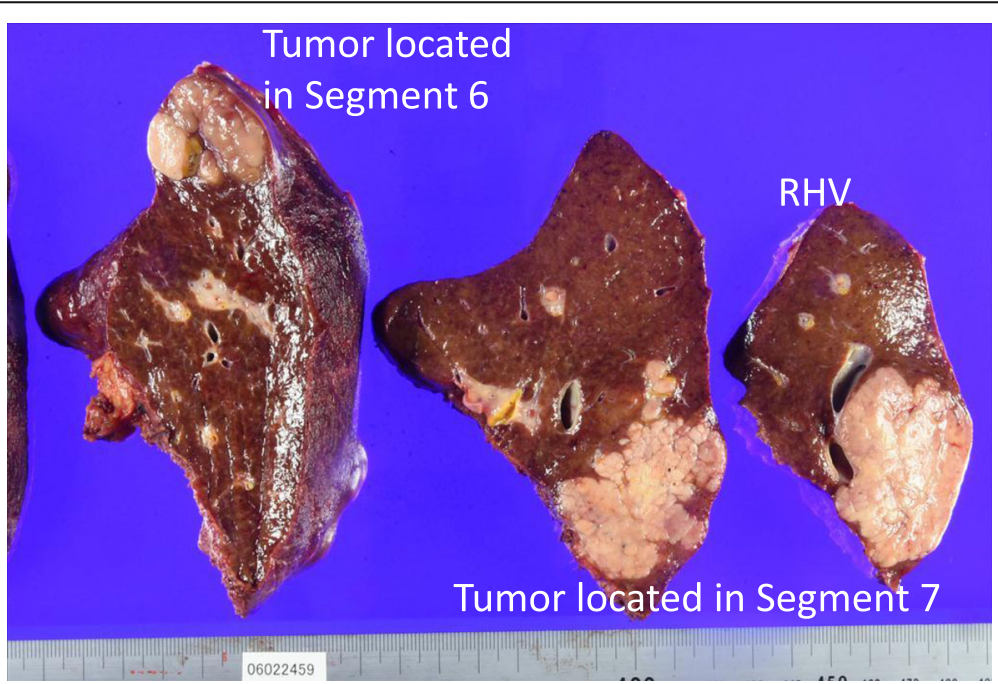

Fig. 5 Macroscopic findings of HCC. Both tumors were confluent, multinodular-type HCC. The right hepatic vein was surrounded by the tumor, but a venous thrombus was not detected. HCC, hepatocellular carcinoma 
large-volume ascites and liver failure in patients with ALICE grade 2 and PH. The ALICE score was calculated by means of the following formula: $0.663 \times \log 10$ ICGR15 - $0.718 \times$ albumin $(\mathrm{g} / \mathrm{dl})$ [12]. The ALICE grade was stratified as follows: ALICE grade 1, linear predictor value of $<-2.20$; ALICE grade $2 \mathrm{a}$, linear predictor value of -2.20 to -1.88 ; ALICE grade $2 \mathrm{~b}$, linear predictor value of -1.88 to -1.39 ; and ALICE grade 3 , linear predictor value of $>-1.39[11,12]$. The ICGR15 has been widely used in the field of hepatobiliary surgery in Japan. Some authors have investigated the usefulness of the ALICE grade to predict liver function or prognosis [11, 12]. Bogner reported that an intraoperative PVP increase was an independent predictor of post-hepatectomy liver failure after major hepatectomy [13]. In our case, PVP was increased by both BRTO and PTPE, the complication after the right lobectomy will be strongly expected. We conclude therefore that we employed an appropriate strategy for treating this patient's HCC with $\mathrm{PH}$ to maximally reduce surgical complications.

Evaluating the degree of liver fibrosis before surgery is also important. Liver biopsy not only is a very high-risk procedure to diagnose liver fibrosis but also has a limited indication. Several reports have focused on the relationship between M2BPGi or VTQ and liver fibrosis. M2BPGi and VTQ were reported to predict liver fibrosis precisely compared with markers such as hyaluronic acid and type IV collagen [14, 15]. According to the cut-off value of M2BPGi and VTQ [14, 15], the fibrosis of these patients could be predicted fibrosis 3 or 4 .

On the other hand, liver fibrosis and portal hypertension after $\mathrm{HCV}$ eradication will improve, but it takes much time [16]. The degree of improvement depends on baseline HVPG. Among patients with HVPG of 10-15 $\mathrm{mmHg}, \mathrm{PH}$ resolved in $43 \%$ after $\mathrm{HCV}$ eradication, but $\mathrm{PH}$ did not resolve among patients with HVPG $\geq$ $16 \mathrm{mmHg}$ [16]. This patient who had risky gastric varices and low platelet count would have tolerated hepatectomy even without splenectomy considering SVR.

Liver transplantation is an ideal treatment for patients with $\mathrm{HCC}$ with $\mathrm{PH}$; however, donor shortages and older recipients mean that candidates for liver transplantation are inevitably limited. Among treatment options, which is best, anatomical or nonanatomical resection? Is synchronous splenectomy better? [17-19]. In any case, modulating portal vein pressure is essential for patients who undergo hepatic resection because PVP is significantly associated with short- and long-term prognoses.

BRTO is a reliable and safe procedure for radical treatment of gastric varices, which are related to portosystemic shunt [20]. BRTO increases hepatopetal portal flow and improves liver function of patients with reserve liver capacity [21]. High splenic volume is an independent predictor of the recurrence of posthepatectomy HCC and overall survival [22]. Thus, splenectomy reduces portal pressure in patients with $\mathrm{PH}$ and improves liver function [23]. Although portal vein thrombosis is a postoperative complication of splenectomy, recent advances in laparoscopic surgery and the treatment of PV thrombosis increase safety and reduce the invasiveness of splenectomy when administered to patients with liver cirrhosis and $\mathrm{PH}$ [24]. Kawanaka et al. found that HVPG significantly increases after B-RTO (average increase, 27.6\%), although HVPG tends to decrease (average reduction, 14.8\%) after BRTO combined with splenectomy [25].

Splenectomy reduces $\mathrm{PH}$, but there were some problems such as portal vein thrombosis and OPSI. Cirrhotic patients after splenectomy show decreased levels of ATIII activity, which are associated with hypercoagulable status, and reduced portal venous flow, resulting from the elimination of increased splenic blood flow. This has been found to amplify the incidence of PVT considerably, as much as 24 to $36 \%$ [26, 27]. Kawanaka et al. reported that ATIII is recommended for patients at high risk for thrombotic complications, including splenectomy in patients with liver cirrhosis, and is safe and effective prophylactic methods that do not increase the risk of bleeding [26]. OPSI is a syndrome of fulminant sepsis occurring in splenectomized patients that are associated with high mortality and morbidity [28]. The pneumococcal vaccination will be mandatory.

To reduce the surgical complication especially such as liver failure or refractory ascites, remnant liver volume is important. PTPE induced significant compensatory hypertrophy whether in the non-cirrhosis group ( $p=$ $0.002)$ or cirrhosis group $(p<0.001)$; however, no significant difference was identified between the two groups, with respect to left liver volume enlargement 46 weeks following PVE $(p=0.373)$ [29]. On the other hand, PTPE needs waiting time for hypertrophy, which allows tumor progression.

Certain interventions for $\mathrm{PH}$ are time-consuming, which allows tumor progression. In the present study, although the levels of the tumor markers AFP and DCP were elevated and tumors were slowly growing, hepatic resection was fortunately performed 10 weeks after the first visit without complications. To measure PVP, WHVP, or HVPG, as performed here, this case may be helpful for indicating hepatic resection. Thus, if PVP before hepatic resection exceeds $15 \mathrm{mmHg}$, partial hepatic resection should be selected.

\section{Conclusions}

Our experience with the present patient who underwent multiple procedures to treat advanced $\mathrm{HCC}$ with $\mathrm{PH}$ provides compelling evidence that controlling PVP is important when the hepatic resection is required for treating this condition. 


\section{Abbreviations}

CT: Computed tomography; CE-CT: Contrast-enhanced CT; US: Ultrasound sonography; MRI: Magnetic resonance imaging; ${ }^{18} \mathrm{~F}-\mathrm{FDG}$-PET: 2-Deoxy-2[fluorine-18]fluoro-D-glucose integrated with computed tomography; ICGR15: Indocyanine green dye retention test at 15 min; HVPG: Hepatic venous pressure gradient; WHVP: Wedged hepatic venous pressure; HCC: Hepatocellular carcinoma; PVE: Portal vein embolization; BRTO: Balloonoccluded retrograde transvenous obliteration; AFP: a-Fetprotein; DCP: des- $\gamma^{-}$ carboxy prothrombin

\section{Acknowledgements}

We thank Edanz Group (www.edanzediting.com/ac) for editing a draft of this manuscript.

\section{Authors' contributions}

$\mathrm{NH}$ reported the case and wrote the manuscript. $\mathrm{RM}, \mathrm{KH}, \mathrm{NI}, \mathrm{MT}$, TI, and NK performed surgery and perioperative management of the patient and helped draft the manuscript. KS and MM performed BRTO and PTPE. KA, HK, and KS participated in revising the manuscript. All authors read and approved the final manuscript.

\section{Funding}

This study was not funded.

\section{Availability of data and materials}

Data sharing is not applicable to this article as no datasets were generated or analyzed during the current study.

\section{Ethics approval and consent to participate}

This case report was performed in accordance with the Declaration of Helsinki, and patients granted their written informed consent to participate in this study according to our institutional consent form.

\section{Consent for publication}

Patients granted their consent to publish their data.

\section{Competing interests}

The authors have no competing interests to declare.

\section{Author details}

${ }^{1}$ Department of Hepatobiliary and Pancreatic Surgery, Graduate School of Medicine, Gunma University, 3-39-22, Showamachi, Maebashi 371-8511, Japan. ${ }^{2}$ Department of Innovative Cancer Immunotherapy, Graduate School of Medicine, Gunma University, Maebashi, Japan. ${ }^{3}$ Department of Radiation Oncology, Graduate School of Medicine, Gunma University, Maebashi, Japan. ${ }^{4}$ Department of Applied Medical Imaging, Graduate School of Medicine, Gunma University, Maebashi, Japan. ${ }^{5}$ Clinical Research Institute and Department of Surgery, National Hospital Organization, Beppu Medical Center, Beppu, Japan.

Received: 20 May 2020 Accepted: 20 July 2020

Published online: 31 July 2020

\section{References}

1. Llovcet JM, Burroughs A, Bruix J. Hepatocellular carcinoma. Lancet. 2003;362: 1907-17.

2. Bruix J, Castells A, Bosch J, Feu F, Fuster J, Garcia-Pagan JC, et al. Surgical resection of hepatocellular carcinoma in cirrhotic patients: prognostic value of preoperative portal pressure. Gastroenterology. 1996;111(4):1018-22.

3. Cucchetti A, Ercolani G, Vivarelli M, Cescon M, Ravaioli M, Ramacciato G, et al. Is portal hypertension a contraindication to hepatic resection? Ann Surg. 2009;250(6):922-8.

4. Llovet JM, Brú C, Bruix J. Prognosis of hepatocellular carcinoma: the BCLC staging classification. Semin Liver Dis. 1999;19(3):329-38.

5. Llovet JM, Fuster J, Bruix J. Intention-to-treat analysis of surgical treatment for early hepatocellular carcinoma: resection versus transplantation. Hepatology. 1999;30(6):1434-40.

6. Takemura N, Aoki T, Hasegawa K, Kaneko J, Arita J, Akamatsu N, et al. Hepatectomy for hepatocellular carcinoma after perioperative management of portal hypertension. Br J Surg. 2019;106(8):1066-74.
7. Tajiri T, Yoshida H, Obara K, Onji M, Kage M, Kitano S, et al. General rules for recording endoscopic findings of esophagogastric varices (2nd edition). Dig Endosc. 2010;22:1-9.

8. Berzigotti A, Reig M, Abraldes JG, Bosch J, Bruix J. Portal hypertension and the outcome of surgery for hepatocellular carcinoma in compensated cirrhosis: a systematic review and meta-analysis. Hepatology. 2015;61(2):526-36.

9. Chen X, Zhai J, Cai X, Zhang Y, Wei L, Shi L, et al. Severity of portal hypertension and prediction of postoperative liver failure after liver resection in patients with Child-Pugh grade A cirrhosis. Br J Surg. 2012; 99(12):1701-10.

10. Hidaka M, Takatsuki M, Soyama A, Tanaka T, Muraoka I, Hara T, et al. Intraoperative portal venous pressure and long-term outcome after curative resection for hepatocellular carcinoma. Br J Surg. 2012;99(9):1284-9.

11. Shirata C, Kokudo T, Arita J, Akamatsu N, Kaneko J, Sakamoto Y. AlbuminIndocyanine Green Evaluation (ALICE) grade combined with portal hypertension to predict post-hepatectomy liver failure. Hepatol Res. 2019; 49(8):942-9.

12. Kokudo T, Hasegawa K, Amikura K, Uldry E, Shirata C, Yamaguchi T, et al. Assessment of preoperative liver function in patients with hepatocellular carcinoma - the Albumin-Indocyanine Green Evaluation (ALICE) grade. PLoS One. 2016;11(7):e0159530.

13. Bogner A, Reissfelder C, Striebel F, Mehrabi A, Ghamarnejad O, Rahbari M, et al. Intraoperative increase of portal venous pressure is an immediate predictor of posthepatectomy liver failure after major hepatectomy: a prospective study. Ann Surg. 2019 in press.

14. Toshima T, Shirabe K, Takeishi K, Motomura T, Mano Y, Uchiyama H, et al. New method for assessing liver fibrosis based on acoustic radiation force impulse: a special reference to the difference between right and left liver. J Gastroenterol. 2011;46(5):705-11.

15. Toshima T, Shirabe K, Ikegami T, Yoshizumi T, Kuno A, Togayachi A, et al. A novel serum marker, glycosylated Wisteria floribunda agglutinin-positive Mac-2 binding protein (WFA(+)-M2BP), for assessing liver fibrosis. J Gastroenterol. 2015;50(1):76-84.

16. Mandorfer M, Kozbial K, Schwabl P, Freissmuth C, Schwarzer R, Stern R, et al. Sustained virologic response to interferon-free therapies ameliorates HCVinduced portal hypertension. J Hepatol. 2016;65(4):692-9.

17. Huang X, Lu S. A Meta-analysis comparing the effect of anatomical resection vs. non-anatomical resection on the long-term outcomes for patients undergoing hepatic resection for hepatocellular carcinoma. HPB (Oxford). 2017;19(10):843-9.

18. Kaibori M, Kon M, Kitawaki T, Kawaura T, Hasegawa K, Kokudo N, et al. Comparison of anatomic and non-anatomic hepatic resection for hepatocellular carcinoma. J Hepatobiliary Pancreat Sci. 2017;24(11):616-26.

19. Kong J, Shen S, Wang W. Synchronous hepatectomy and splenectomy vs hepatectomy for selected patients with hepatocellular carcinoma and clinically significant portal hypertension: a systematic review and metaanalysis. J Surg Oncol. 2019;119(7):964-73.

20. Kawanaka H. Balloon-occluded retrograde transvenous obliteration: one step beyond obliteration of gastric varices. J Gastroenterol Hepatol. 2012; 27(1):3-4.

21. Uehara H, Akahoshi T, Tomikawa M, Kinjo N, Hashimoto N, Nagao Y, et al Prediction of improved liver function after balloon-occluded retrograde transvenous obliteration: relation to hepatic vein pressure gradient. J Gastroenterol Hepatol. 2012;27(1):137-41.

22. Takeishi K, Kawanaka H, Itoh S, Harimoto N, Ikegami T, Yoshizumi T, et al. Impact of splenic volume and splenectomy on prognosis of hepatocellular carcinoma within milan criteria after curative hepatectomy. World J Surg. 2018;42(4):1120-8.

23. Kawanaka $H$, Akahoshi $T$, Ito $S$, Iguchi T, Harimoto $N$, Uchiyama $H$, et al. Optimizing risk stratification in portal vein thrombosis after splenectomy and its primary prophylaxis with antithrombin III concentrates and danaparoid sodium in liver cirrhosis with portal hypertension. J Am Coll Surg. 2014;219:865-74.

24. Kawanaka H, Akahoshi T, Kinjo N, Konishi K, Yoshida D, Anegawa G, et al. Technical standardization of laparoscopic splenectomy harmonized with hand-assisted laparoscopic surgery for patients with liver cirrhosis and hypersplenism. J Hepatobiliary Pancreat Surg. 2009;16:749-57.

25. Kawanaka H, Akahoshi T, Nagao Y, Kinjo N, Yoshida D, Matsumoto Y, et al. Customization of laparoscopic gastric devascularization and splenectomy for gastric varices based on CT vascular anatomy. Surg Endosc. 2018;32(1): $114-26$. 
26. Kawanaka H, Akahoshi T, Kinjo N, Konishi K, Yoshida D, Anegawa GO, et al Impact of antithrombin III concentrates on portal vein thrombosis after splenectomy in patients with liver cirrhosis and hypersplenism. Ann Surg. 2010;251(1):76-83.

27. Kinjo N, Kawanaka H, Akahoshi T, Tomikawa M, Yamashita N, Konishi K, et al. Risk factors for portal venous thrombosis after splenectomy in patients with cirrhosis and portal hypertension. Br J Surg. 2010;97(6):910-6.

28. Tahir F, Ahmed J, Malik F. Post-splenectomy sepsis: a review of the literature. Cureus. 2020;12(2):e6898.

29. Sun JH, Zhang YL, Nie CH, Li J, Zhou TY, Zhou GH, Zhu TY, Chen LM, Wang WL, Zheng SS. Effects of liver cirrhosis on portal vein embolization prior to right hepatectomy in patients with primary liver cancer. Oncol Lett. 2018; 15(2):1411-6.

\section{Publisher's Note}

Springer Nature remains neutral with regard to jurisdictional claims in published maps and institutional affiliations.

\section{Submit your manuscript to a SpringerOpen ${ }^{\circ}$ journal and benefit from:}

- Convenient online submission

- Rigorous peer review

- Open access: articles freely available online

- High visibility within the field

- Retaining the copyright to your article

Submit your next manuscript at $\boldsymbol{\nabla}$ springeropen.com 